\title{
Guidance for paediatric emergency departments/rooms and departments of paediatrics on the management of a child suspected of or diagnosed with COVID-19
}

\author{
Teresa Jackowska', Magda Marczyńska², Jarosław Peregud-Pogorzelski ${ }^{3}$ \\ 'National Consultant in the field of Paediatrics, Polish Paediatric Society, Department of Paediatrics, Medical Centre \\ of Postgraduate Education, Warsaw, Poland \\ ${ }^{2}$ President of the Expert Advisory Panel for Children Suspected or Infected with SARS-CoV-2 of the National Consultant \\ in the field of Infectious Diseases, Department of Paediatric Infectious Diseases, Medical University of Warsaw, Warsaw, \\ Poland \\ ${ }^{3}$ President of the Polish Paediatric Society, Department of Paediatrics, Paediatric Oncology, and Immunology, Pomeranian \\ Medical University, Szczecin, Poland
}

\section{ABSTRACT}

The presented guidance describes the basic principles of the management of children admitted to the emergency department (ED), emergency room (ER), or hospital diagnosed or not with COVID-19. This guidance is based on the literature review conducted and is valid at the date of issue. It may evolve over time as our knowledge about the infection increases. The majority of children will develop mild symptoms from the infection. In addition, the majority of children will not have any breathing problems.

Current experience suggests that the department of paediatrics will not be involved in the frontline fight against coronavirus. However, we will play a crucial role in that fight, and so we should remember and plan our actions well.

\section{KEY WORDS:}

COVID-19, SARS-CoV-2, guidance.

The Chinese city of Wuhan is where coronavirus disease 2019 (COVID-19), which is caused by a novel coronavirus (SARS-CoV-2), was discovered for the first time [1]. Although the Chinese government quickly took the appropriate measures to limit the movement of people, the virus rapidly spread to other provinces $[2,3]$. On 28 April 2020 China had reported 83,938 cases and 4,637 deaths (https://www.ecdc.europa.eu/en/geographical-distribution-2019-ncov-cases [4]). COVID-19 has become a serious global public health issue. As of 28 April 2020, $\mathbf{2 , 9 8 2 , 6 8 8}$ cases of COVID-19 have been reported in the world, including $\mathbf{2 1 0 , 1 9 3}$ deaths; $1,087,272$ cases have been reported in the European Union/European Economic Area (EU/EEA) and the United Kingdom (UK), and 944,116 people have been reported to have recovered. The largest number of COVID-19 cases has been diagnosed in the United States (US). In Poland, there have been 12,219 confirmed cases of infection and 596 deaths; 2,635 people have recovered (as of 28/04/2020), giving an incidence of 31.33 per 100,000 inhabitants (data from the Ministry of Health [MZ] and European Centre for Disease Prevention and Control [ECDC]) [4].

SARS-CoV-2 infection, diagnosed in 2002, can induce a severe disease of the respiratory system similar to the

\section{ADDRESS FOR CORRESPONDENCE:}

Teresa Jackowska, Department of Paediatrics, Medical Centre of Postgraduate Education, 99/103 Marymoncka St., 01-813 Warsaw, Poland, ORCID: 0000-0003-0131-7854, e-mail: tjackowska@cmkp.edu.pl 
coronavirus (SARS-CoV), causing severe acute respiratory syndrome (SARS) $[5,6]$. The symptoms of COVID-19 caused by SARS-CoV-2 include fever, cough, myalgia, or fatigue as well as the recently added headache, and smell and taste disorders [5]. It was demonstrated that SARS$\mathrm{CoV}-2$ is transmitted between people via direct contact and through the inhalation of airborne droplets [7-9] as well as via contact with surfaces contaminated with the virus.

Children can present similar symptoms of COVID-19 as adults; however, in the paediatric population, the symptoms are more often milder and include a fever below $39^{\circ} \mathrm{C}$, running nose, cough, and sore throat; vomiting and diarrhoea occur rarely. Moreover, radiological or laboratory signs of infection are also less frequent. Furthermore, children diagnosed with COVID-19 can be completely asymptomatic and not require any medical assistance [10-13].

The chest X-ray of children with COVID-19 can be normal or present uni- or bilateral heterogeneous opacities or interstitial consolidations. Chest X-rays and/or CT scans can reveal ground-glass opacities that do not always correlate with the severe clinical condition of the patient. Pleural reaction or mediastinal lymph node enlargement are not typical signs of COVID-19.

Moreover, laboratory tests such as CBC, levels of infection biomarkers, coagulation factors, and biochemistry can all be normal. The characteristic COVID-19-related abnormalities include an increase in the level of an acute phase protein (CRP) accompanied by a normal procalcitonin level. The factors associated with a more severe course of the disease include an increase in the level of lactate dehydrogenase (LDH), fibrinogen and D-dimers, interleukin (IL)-6, ferritin, CRP; progressive leukopenia with granulocytosis and lymphocytopaenia; as well as abnormalities indicating internal organ damage.

In children, viral excretion in the gastrointestinal (GI) tract can be higher and longer compared to the respiratory tract. However, the ability of the virus excreted in faeces to replicate has not been studied.

It should be noted that, in the majority of paediatric cases of COVID-19, the suspicion of infection was made based on the medical interview/history of contact (most often with a family member) with an adult diagnosed with COVID-19 [4].

It is vital to mention the possibility of presymptomatic SARS-CoV-2 transmission, which makes disease control challenging. Seven cases of SARS-CoV-2 transmission from a presymptomatic infected person to other people were reported in Singapore. Therefore, a presymptomatic SARS-CoV-2 transmission was highly probable in those cases. Non-specific prevention measures are of crucial importance in controlling the COVID-19 pandemic. They include adhering to the basic rules of hygiene (handwashing), covering the face, and maintaining distance (2 meters) between persons [14].
On 11 March 2020, the World Health Organization (WHO) announced the pandemic of SARS-CoV-2, the virus that causes COVID-19 [15]. Two days later, on 13 March 2020, the government of the Republic of Poland announced the national state of epidemic emergency [16]. On 20 March 2020, the state of the epidemic, based on the regulation of the Minister of Health, was enforced in the Republic of Poland [17].

In the following four main points and several essential steps, we present the procedure for emergency departments (ED), paediatric emergency rooms (ER), and departments of paediatrics on the management of a child suspected of or diagnosed with COVID-19 [18-23].

\section{MANAGEMENT OF A CHILD VISITING THE EMERGENCY DEPARTMENT/EMERGENCY ROOM DURING THE COVID-19 PANDEMIC}

\section{STEP 1.}

Before coming into contact with healthcare workers (before entering the ED/paediatric ER [PER]), the patient or their parent(s) should be interviewed via hospital phone/intercom.

1. Phone interview regarding COVID-19 symptoms in a child before entering the ED/PER:

1.1. What symptoms does the child have?
a. cough
yes/no
b. fever (body temperature above $38^{\circ} \mathrm{C}$ ) yes $/$ no
c. shortness of breath/respiratory problems yes/no
d. other symptoms? If so, ask what the symptoms are. Document the symptoms in the medical re- cords: telephone triage
e. transported by medical transport yes/no
f. with/without referral diagnosis
g. mode of admission emergency/scheduled

1.2 Within the last 14 days, has the child had direct contact with a:
a. person diagnosed with COVID-19
yes/no
b. person suspected of COVID-19
c. person who was in isolation/under quarantine
yes/no yes/no

\section{STEP 2.}

Interpretation of the information received via the telephone interview and the determination of the place of consultation:

1. At a minimum, if one of the symptoms listed in point $1.1(a-c)$ is present or the answer to any of the questions asked in point 1.2 is YES, the child is placed in an isolation room intended for a child suspected of COVID-19 (see below - information about personal protective equipment [PPE] for healthcare personnel).

2. A multi-speciality hospital must have a separate patient reception desk for children, including children 
suspected of COVID-19; e.g. half of the ED/PER or a medical tent provided by firefighters. There is a need to label the communication lane to avoid crossing different patient pathways.

3. A paediatric hospital requires a separate patient reception desk intended for children suspected of COVID-19.

4. ATTENTION! A child with COVID-19 can come to any ED/PER or department of paediatrics in the country!

Parents might provide confusing, false, or incomplete information.

Parents may not have any information about the contact of their child with COVID-19.

Every child arriving at the ED/PER should be treated as a child suspected of COVID-19.

All epidemiological procedures should be increased during the pandemic.

The current recommendations of the Chief Sanitary Inspectorate (GIS) and its subordinate structures should be followed.

The actual recommendations of the Infection Control Team (ICT) should be followed. Recommendations developed for the ED/PER and departments of paediatrics can differ from the recommendations followed at the ED for adults.

\section{STEP 3.}

Child suspected of COVID-19 based on the information obtained via a phone call:

1. If the answer to any question asked in point 1.2 is YES (STEP 1), and the child does not have any co-morbidities or has a chronic disease (previously diagnosed; is treated for chronic diseases), but currently does not require specialised care, the patient is to be transferred to a designated infectious diseases hospital dedicated to treating children with COVID-19 - phone contact with a doctor is required (information available on the website of the Regional Consultant in the field of paediatrics, the local division of the Polish Paediatric Society [PTP], and on the website of the PTP) ${ }^{1}$.

2. If a child is admitted to the ED-PER, a medical admissions clerk/nurse shall give a questionnaire (annexe 1) to the carer and indicate an isolation room/place in the ED/PER where the patient should go. The carer completes the questionnaire.

3. A nurse (dressed in a disposable fleece gown, surgical mask, face shield, and protective gloves) performs triage within 15 minutes: measurement of the child's respiratory rate, oxygen saturation, and body temperature. Next, they take the completed annexe 1 from a carer and then leave the ED/PER. They complete a nursing assessment form (annexe 2). They pass both annexes ( 1 and 2$)$ to the PER/ED doctor ${ }^{2}$.

4. The patient is registered based on the information from annexe 1 .

5. When the suspicion of COVID-19 is questionable and the patient presents symptoms potentially indicating other causes of the disease, an on-site nasopharyngeal swab specimen for SARS-CoV-2 detection by RT-PCR should be taken; other tests should be performed depending on the child's clinical condition assessment.

6. Attention! Collecting a nasopharyngeal swab (procedure with a high risk of infection): the nurse must be dressed in PPE to achieve respiratory isolation:

a. water-resistant gown,

b. double protective gloves,

c. face mask with an FFP2 or FFP3 filter,

d. face shield,

e. hair cap.

Using one swab (limited availability of swabs), perform a deep swab from the nasopharynx and both nasal ducts. Place the swab in a collection tube. Break the swab at the groove and discard what remains of the swab (if too long). Close the collection tube. Insert the tube into a plastic biohazard bag held by an assistant. If the specimen is sent outside the facility, follow the specified rules for the transport of biological material to the laboratory.

Inform the ICT (or other person designated by the management of the hospital) and the Sanitary-Epidemiological Station (SANEPID) about the specimen collection.

\section{STEP 4.}

Management after receiving the result of the SARSCoV-2 RT-PCR test:

1. Negative result - communicate the test result to the patient, ICT, and SANEPID. If the patient is in good health condition and does not require hospitalisation, they should be discharged home. Remember! If the family had contact with a COVID-19-positive patient, the patient, along with their carer, should stay under home quarantine for 14 days from the date of contact.

2. Positive result - inform the patient. The doctor decides if the patient needs to be admitted to the designated infectious diseases hospital dedicated to children with COVID-19 or to a specialist hospital (if indicated) dedicated to patients with COVID-19. The hospital should be informed earlier by phone about the patient transfer. Inform the ICT and SANEPID. 


\section{STEP 5.}

1. In cases previously diagnosed with COVID-19 (without repeating the nasopharyngeal swab), a physician decides if the patient needs to be admitted to one of the following:

a. a designated infectious diseases hospital dedicated to children with COVID-19,

b. a specialist hospital (if the child requires the care of a specialist) dedicated to children with COVID-19,

c. a designated paediatric hospital with an intensive care unit (ICU) when the patient's condition is life-threatening, and they require intensive care.

Remember! Call for an ambulance intended to transport COVID-19 patients.

2. If a child suspected of COVID-19 is admitted to the hospital to which they have come, they should stay in an isolation room intended for a child suspect of COVID-19 until the moment of transfer to the designated infectious diseases department or a specialist department dedicated to the COVID-19 patient, or until obtaining the results of the COVID-19 test.

3. Personal protective equipment (PPE) for respiratory isolation intended for healthcare workers working in the ED/PER during the contact with a child suspected of COVID-19. A healthcare worker should put on the following equipment before contacting the patient:

a. face mask with FFP2 or FFP3 filter (4-hour protection),

b. face shield,

c. hair cap,

d. gloves,

e. fleece gown or water-resistant gown when invasive procedures are needed.

4. Personal protective equipment (PPE) for the patient and carer:

a. surgical mask during transport, transfer for medical examinations/tests, or during contact with other persons,

b. need to isolate the patient in a single patient room (if not previously done, it is urgently required that from 1 to 3 isolation rooms are separated depending on the needs and capacities!).

\section{STEP 6.}

When the diagnosis of diseases other than COVID-19 is uncertain, blood and urine samples for testing; perform an ultrasound and radiological examination in the ED/ PER (doctor's individual decision) in order to determine the need for a child's hospitalisation for reasons other than COVID-19; or admission of a child to an appropriate department of paediatrics in the given hospital (when COVID-19 is ruled out based on the medical interview and a nasopharyngeal swab!). The child's length of stay (LOS) during the pandemic should be reduced to a minimum.
STEP 7.

No indications for the hospitalisation of a child without suspicion of COVID-19: the child is discharged home; the patient receives a hospital discharge summary report (HDSR) from the ED/PER with medical recommendations; it is necessary to inform the doctor working in the outpatient clinic about the patient's visit to the ED/PER, and to provide them with the patient's HDSR.

STEP 8.

When a patient suspected of or diagnosed with COVID-19 leaves the room (ED/PER/isolation room), the following recommendations apply:

1. Carry out no-touch disinfection with the use of fogging with hydrogen peroxide or ultraviolet light (UV-C) for a time adjusted to the room's volume, if possible. After using hydrogen peroxide, the room should be aerated.

2. After the disinfection phase mentioned above, the room should be cleaned with the use of alcohol- (minimum alcohol content of 70\%) or chlorine-based products (minimum concentration of 1000-2000 ppm); all surfaces potentially touched by the patient (handles, bed/chair handrails, taps) should be disinfected. Caution! The use of disinfectant sprays can create aerosols containing infectious particles that lift off from disinfected surfaces and float in the air thereby becoming a potential threat to the person cleaning the room; thus, it is not recommended. Surfaces should be disinfected with the use of impregnated cleaning wipes or cloths.

3. Remove the disposable fleece sheet and put on a new one.

\section{STEP 9. REMEMBER!}

1. Every child arriving at the ED/PER needs to be examined (except for STEP 1, point 1.2). A child can be transferred with the use of own-account transport or an ambulance intended for the transportation of COVID-19 patients.

2. The management depends on the child's general health condition.

3. Children often have a fever and cough for many other reasons not associated with COVID-19.

4. The COVID-19 pandemic does not exclude the occurrence of other diseases (including cancer or sepsis).

5. Not every child suspected of COVID-19 requires hospitalisation.

6. Children with COVID-19 can have no or atypical symptoms.

\section{STEP 10. SUMMARY}

1. If a child in the ED/PER presents with symptoms indicating any disease requiring hospital treatment, the 
eventual association of those symptoms with SARS$\mathrm{CoV}-2$ infection is unlikely.

2. In case of any doubts, admit the child to the hospital; collect a nasopharyngeal swab for SARS-CoV-2 infection and then treat the disease that has been diagnosed.

3. Pending the result of the test, consider the child as a child suspected of COVID-19 (management as above).

4. If a child's carer did not have any contact with a person infected with SARS-CoV-2 and is healthy, the child, after receiving a negative test result, should not be isolated.

5. Remember that departments of paediatric infectious diseases are intended during the pandemic to treat children with COVID-19. Patients with other infectious diseases should be treated in the department of paediatrics.

\section{MANAGEMENT OF A CHILD IN THE DEPARTMENT OF PAEDIATRICS DURING THE COVID-19 PANDEMIC}

\section{STEP 1.}

Transfer of a patient suspected of COVID-19 from the ED/PER to the department:

1. The patient, accompanied by one carer (both wearing surgical masks!), is escorted to the department by a healthcare worker wearing a surgical mask, a fleece gown, and gloves.

2. The patient and their carer are immediately directed to the isolation room as previously agreed by phone. During this time, a healthcare worker working in the department keeps a distance of at least two metres from the patient and their carer (they should, preferably, follow the healthcare worker to the isolation room).

\section{STEP 2.}

Hospitalisation of a child suspected of COVID-19 respiratory isolation is required until receiving the test results (see what PPE should be used):

1. Evaluate the patient's general health condition (measurement of oxygen saturation, respiratory rate, body temperature, heart rate, and pulse are necessary).

2. Examine the patient.

3. Collect the patient's material samples for tests in line with a primary diagnosis (tests selected based on a doctor's decision).

4. When COVID-19 is suspected, collect samples for COVID-19 testing (in line with the instructions, collect samples for tests determined by experts in the field of paediatrics related to COVID-19 pandemic). A nurse (dressed in PPE) enters the isolation room with a tray containing gauze pads, disinfectants, peripheral venous lines, dressings, disposable tourniquet, thermometer, infusion fluid, and medications ordered by the doctor. It is forbidden to take the cart into the isolation room. A pneumatic tube should not be used during the transport of the patient's samples to the laboratory.

5. Do not order tests performed outside the child's room if they are not needed; do not use the reusable equipment.

6. Minimise the number of aerosol-generating procedures (AGP), such as nebulisation; administer drugs through an inhalation chamber. If a child requires oxygen therapy, administer oxygen through a face mask.

7. If systemic drugs are needed, the enteral route of administration, instead of intravenous (IV), is preferred.

8. Minimise the number of entries into the isolation room.

9. Patient observation forms (for controlling body temperature, oxygen saturation, fluid intake balance, and ailments) (annexe 3) filled in by the parent/carer should be located in an easily visible place in the isolation room.

10. The parent/carer informs the healthcare worker about the child's condition four times a day. In the case of the presence of any disturbing symptoms, the parent/ carer should immediately inform the healthcare worker by phone (from their phone).

11. The parent/carer should receive information about the rules of isolation; the risk of contracting the infection from their child; ban on leaving the room and for visiting the patient/parent); and the phone number of a nurse/doctor should be available in a visible place.

12. The patient and their parent/carer should receive bottled water and disposable cups (separately for the patient and their carer).

13. Meals for a child and their carer are left on the table placed in front of the entrance to the isolation room; the carer takes the meal when there are no healthcare workers in the proximity. A notification system should be established.

14. The isolation room (if not equipped with negative pressure) should be regularly aerated!

\section{STEP 3. REMEMBER!}

1. In the majority of cases, the hospitalised child will have a disease other than COVID-19.

2 . If a child presents symptoms indicating any disease requiring hospitalisation, an eventual association of those symptoms with SARS-CoV-2 infection is unlikely.

3. In case of any doubts, collect a nasopharyngeal swab for SARS-CoV-2 infection, and treat the disease that you have diagnosed.

4. Regarding similar symptoms to COVID-19, influenza/ RSV infection should be ruled out during the period of seasonal influenza/RSV infections (Remember! A positive test result does not rule out COVID-19, but markedly reduces the probability of the disease presence). 
5. Pending the result of the test, consider the child as a child suspected of COVID-19.

\section{STEP 4.}

1. Test for COVID-19 is positive: transfer the child (by prior phone arrangement) to a designated hospital/department of infectious diseases (see recommendations issued by the voivode, the regional consultant in the field of paediatrics).

2. Attention! In Poland, the incidence of COVID-19 in children is relatively low. Data on the course of the disease suggest that paediatric patients more often present milder symptoms compared to adults, and rarely require hospitalisation. Patients in good general health condition can potentially stay at home; however, they should be in constant phone contact with a doctor experienced in treating children with COVID-19 or with a doctor working in the outpatient clinic.

3. Test for COVID-19 is negative: the child does not require isolation. Remember! The length of the hospital stay during a pandemic should be reduced to the minimum! If the child is in stable good general health condition, they should be discharged home; give the hospital discharge summary report (HSDR) with medical recommendations; recommend the need to notify the doctor working in the outpatient clinic about the child's hospitalisation/pass the HSDR; arrange the follow-up visit in a paediatric (up to 90 days after discharge) or specialist outpatient clinic (inform the patient/carer that the visit can be carried out by phone), when necessary.

\section{RECOMMENDATIONS FOR PARENTS/ CARERS STAYING TOGETHER WITH A CHILD DURING THE COVID-19 PANDEMIC}

\section{The parent/carer can always stay with their child} in the department of paediatrics (general, specialist), either when the diagnosis of COVID-19 is ruled out, suspected, or confirmed.

2. The child should stay with one, healthy carer. If both parents are infected (SARS-CoV-2), the child stays with a carer not requiring hospitalisation. If both carers require hospitalisation, they can designate a substitute carer (not grandparents - increased risk of COVID-19 development!) or a carer will be provided by the department's healthcare workers.

3. A doctor/nurse should inform the parent/carer (consent obtained on admission or by phone) about the:

a. ban on leaving the isolation room, patient room (concerns all children hospitalised during the pandemic),

b. rules of isolation,

c. risk of contracting the infection from their child,

d. ban on external visits, e. method of contact (preferably by phone) with healthcare workers; always put the phone number of the nurse/doctor in a visible place.

4. The carer should manage the self-assessment (a daily measurement of the body temperature and an evaluation of the symptoms). If the carer falls sick or presents any symptoms, they should be transferred to the ED/ ER of a hospital for adults or should undergo nasopharyngeal swab for SARS-CoV-2 RT-PCR; the SANEPID should be notified. When the carer tests positive for SARS-CoV-2 infection and the child does not have an infection, they should be transferred for isolation; in addition, the second carer of that child should be informed about the situation. The child with their new/ second carer stays in the hospital requiring respiratory isolation - quarantine.

5. During hospitalisation, meals and tea, as well as drinking water for the parent/carer, are provided by the hospital.

6. When COVID-19 is suspected/diagnosed, meals are left on a table placed in front of the entrance of the isolation room; the carer takes the meal when there are no healthcare workers in the proximity. Also, a notification system should be established.

7. Recommendations on breastfeeding are in line with the guidance issued by the National Consultant in the field of neonatology that was published in cooperation with other scientific associations.

8. A hospitalised infant with SARS-CoV-2 infection can be breastfed.

9. During hospitalisation, cleaning products and diapers are provided by the hospital.

IV. RECOMMENDATIONS LIMITING THE RISK OF SARS-COV-2 TRANSMISSION AMONG THE HEALTHCARE WORKERS OF THE DEPARTMENTS OF PAEDIATRIC EMERGENCY DEPARTMENTS/PAEDIATRIC EMERGENCY ROOMS

\section{A. CONTACT WITH A PATIENT/CHILD UNTIL RULING OUT THE INFECTION}

1. FFP2/3 face mask (protection time up to 4 hours) or a surgical mask (protection time up to 15 minutes) is required.

2. Eye protection (goggles, face shield) is needed.

3. The use of PPE during each direct contact with the patient in hospital conditions is necessary:

a. when entering the room, the patient is always asked to put their face mask on, if possible,

b. when entering the room (for up to 15 minutes), a regular surgical mask, long-sleeved gown, protective gloves, and eye protection are sufficient,

c. in close contact with the patient (less than 2 metres), blood draws, ENT examination; during aero- 
sol-generating procedures as well as hygiene-care activities (bath, changing diapers), FFP2/3 mask (surgical mask only when a filtered face mask is unavailable), face shield, protective gloves, and a water-resistant gown are required,

4. Minimisation of healthcare resources use: one FFP $2 / 3$ mask can be used for up to four hours; there is no need to change the mask between two patients with potential COVID-19 (when there was no contact with the contaminated surface of the mask or until the patient did not cough [on the mask]). The face shield protects against such contamination of the face mask. When using goggles, it is possible to protect the filtered face mask by covering it with a disposable surgical mask.

5. The length of time while in contact with the patient should be reduced to the minimum. Medical interview, all discussions, including during hospitalisation, should be carried out by phone. The patient should be asked to prepare for the examination also by phone.

6. The number of doctor's orders should be reduced to the minimum, enabling the shortest length of time of realisation (e.g. antibiotic administration 1-2 instead of 3 times a day, if possible).

7. The working time of healthcare workers should be reduced to the minimum regardless of the actual working hours. It is indicated to follow a rotating shift pattern, teamwork, and weekly shifts. Such management allows one part of the personnel to remain in the hospital at a time, while the other part can work remotely (to answer patients' phone calls or to carry out other orders of the hospital management, head of the department, or immediate superior). Members of the teams should not come into contact with each other, which will allow for putting only one team under quarantine, when necessary. That is crucial, especially for the need to consult or treat sick children who also have medical conditions other than COVID-19.

8. Recommended methods of work in hospital: onetime or limited to a necessary minimum examination of patients, the mandatory keeping of medical records. Consultations and examinations of patients during the pandemic should be carried out by a specialist from only one of the designated teams; only one attending physician, on-duty doctor, when possible.

9. During the pandemic, the doctor examining a child diagnosed with or suspected of COVID-19, until receiving the test result, dresses in and undresses from PPE in line with the recommendations of the ECDC. Doctor/nurse dresses in PPE before entering the isolation room with a sick child. However, they undress from the PPE after leaving the isolation room in another place designated for that purpose, strictly following all the safety principles. When examining subsequent children diagnosed with or suspected of COVID-19, the doctor does not change the face mask (FFP2/3), gown (water-resistant), or shield face, except for outer protective gloves. Always follow the rules of correct undressing from PPE.

10.During the pandemic, the completing of time-consuming parts of medical records should be limited: minimisation of the number of forms, especially documents completed/signed by the patient.

11.Limiting/avoiding the number of outpatient follow-up visits is necessary (does not refer to the follow-up visits after visiting the ED/PER) - phone contact with the doctor should be arranged.

\section{B. CONTACT BETWEEN HEALTH CARE WORKERS}

1. Employee meetings (morning briefings, social meetings) should be banned or limited. Information about the patients is passed on in a quick manner or by phone/e-mail.

2. Workplace organisation - the following are necessary:

a. limit the number of doctors/nurses in doctors'/nurses' offices,

b. ban doctors from moving freely between rooms, hospital departments, offices,

c. visits and consultations between departments should be limited to the necessary minimum; they should be carried out by phone or on-line, if possible,

d. limit the time that healthcare workers stay in the common areas; we do not mix common areas for different groups of healthcare workers; we do not freely move between hospital departments; we do not use common hospital areas, such as buffets, canteens, or restaurants, which should remain closed during the pandemic.

\section{DISCLOSURE}

The authors declare no conflict of interest.

\section{REFERENCES}

1. Lu H, Stratton CW, Tang YW. Outbreak of Pneumonia of Unknown Etiology in Wuhan China: The Mystery and the Miracle. J Med Virol 2020; 92: 401-402.

2. Du Z, Wang L, Cauchemez S, et al. Risk for transportation of 2019 novel coronavirus disease from Wuhan to other cities in China. Emerg Infect Dis 2020; 26: 1049-1052.

3. Kupferschmidt K, Cohen J. Can China's COVID-19 strategy work elsewhere? Science 2020; 367: 1061-1062.

4. https://www.worldometers.info/coronavirus/ (access: 1.04.2020).

5. Huang C, Wang Y, Li X, et al. Clinical features of patients infected with 2019 novel coronavirus in Wuhan, China. Lancet 2020; 395: 497-506.

6. Xu Z, Shi L, Wang Y, et al. Pathological findings of COVID-19 associated with acute respiratory distress syndrome. Lancet Respir Med 2020; 8: 420-422.

7. Chan JFW, Yuan S, Kok KH, et al. A familial cluster of pneumonia associated with the 2019 novel coronavirus indicating per- 
son-to-person transmission: a study of a family cluster. Lancet 2020 395: 514-523

8. Li Q, Guan X, Wu P, et al. Early transmission dynamics in Wuhan, China, of novel coronavirus-infected pneumonia. N Engl J Med 2020; 382: 1199-1207.

9. Wang D, Hu B, Hu C, et al. Clinical characteristics of 138 hospitalised patients with 2019 novel coronavirus-infected pneumonia in Wuhan, China. JAMA 2020; 323: 1061-106.

10. Xu Y, Li X, Zhu B, et al. Characteristics of pediatric SARS-CoV-2 infection and potential evidence for persistent fecal viral shedding. Nat Med 2020; 26: 502-505.

11. Wei M, Yuan J, Liu Y, et al. Novel Coronavirus Infection in Hospitalised Infants Under 1 Year of Age in China. JAMA 2020; 323: 1313-1314.

12. Xia W, Shao J, Guo Y, et al. Clinical and CT features in pediatric patients with COVID-19 infection: Different points from adults. Pediatr Pulmonol 2020; 55: 1169-1174.

13. Chang D, Lin M, Wei L et al. Epidemiologic and Clinical Characteristics of Novel Coronavirus Infections Involving 13 Patients Outside Wuhan, China. JAMA 2020; 323: 1092-1093.

14. Wei WE, Li Z, Chiew CJ, at al. Presymptomatic Transmission of SARS-CoV-2 - Singapore, 23 January-16 March 2020. MMWR Morb Mortal Wkly Rep 2020; 69: 411-415.

15. Sohrabi C, Alsafi Z, O'Neill N, et al. World Health Organization declares global emergency: A review of the 2019 novel coronavirus (COVID-19). Int J Surg 2020; 76: 71-76.

16. Rozporządzenie Ministra Zdrowia z dnia 13 marca 2020 r. w sprawie ogłoszenia na obszarze Rzeczypospolitej Polskiej stanu zagrożenia epidemicznego (Dz.U. z 2020 r. poz. 433).

17. Rozporządzenie Ministra Zdrowia z dnia 20 marca 2020 r. w sprawie ogłoszenia na obszarze Rzeczypospolitej Polskiej stanu epidemii (Dz.U. z 2020 r. poz. 491).

18. Sinha I, Alder Hey Children's Hospital. Guidance for the clinical management of children admitted to hospital with proven COVID-19. https://alderhey.nhs.uk/application/files/1815/8422/1173/ COVID_19_IN_CHILDREN_CLINICAL_GUIDANCE_v1.pdf. (access: 28.03-1.04.2020).

19. Clinical guide for the management of paediatric patients during the coronavirus pandemic. 16 March 2020, Version 1. https://www. england.nhs.uk/coronavirus/wp-content/uploads/sites/52/2020/03/ Specialty-guide_paediatrics-and-coronavirus_V1_17-March.pdf (access: 28.03-1.04.2020).

20. https://services.aap.org/en/pages/2019-novel-coronavirus-covid-19-infections/ (access: 28.03-1.04.2020).

21. https://www.cdc.gov/coronavirus/2019-ncov/hcp/caring-for-patients.html (access: 28.03-1.04.2020)

22. https://www.cdc.gov/coronavirus/2019-ncov/infection-control/control-recommendations.html (access: 28.03-1.04.2020).

23. https://redbook.solutions.aap.org/ss/rbo_outbreaks_page_3.aspx (access: 28.03-1.04.2020) 